\title{
Transthoracic echocardiographic and clinical predictors in first versus recurrent ischemic strokes
}

\author{
Yasser Bahnacy $^{*}$, Amr Abo Koora ${ }^{2}$, Hamed Dawoud ${ }^{3}$, Wafa Rashed ${ }^{1}$, Ali Sadek ${ }^{4}$ \\ ${ }^{1}$ Cardiology Division, Department of Medicine, Mubarak Al-Kabeer Hospital, Hawally, Kuwait \\ ${ }^{2}$ Neurology Division Department of Medicine, Mubarak Al-Kabeer Hospita, Hawally, Kuwait \\ ${ }^{3}$ Cardiology Division, Chest Diseases Hospital, Ferwania, Kuwait \\ ${ }^{4}$ Public Health Department, Ministry of Health, Ferwania, Kuwait \\ Email: *yasserbahnacy@hotmail.com, „ybahnacy@yahoo.com, qoora2004@yahoo.com, hameddawoud@hotmail.com, \\ dr.wrashed@gmail.com, ali_sadek@yahoo.com
}

Received 31 December 2013; revised 5 February 2014; accepted 12 February 2014

Copyright (C) 2014 Yasser Bahnacy et al. This is an open access article distributed under the Creative Commons Attribution License, which permits unrestricted use, distribution, and reproduction in any medium, provided the original work is properly cited. In accordance of the Creative Commons Attribution License all Copyrights (C) 2014 are reserved for SCIRP and the owner of the intellectual property Yasser Bahnacy et al. All Copyright (C) 2014 are guarded by law and by SCIRP as a guardian.

\section{ABSTRACT}

Recurrent strokes are more likely to be disabling or fatal than first ever strokes. The aim of the study is to evaluate transthoracic echocardiographic (TTE) and clinical predictors in patients with first versus those with recurrent ischemic strokes. A prospective observational comparative study of 217 patients admitted with ischemic strokes who were in sinus rhythm. Two groups of patients were simultaneously enrolled. The first group was 110 patients with first ischemic stroke and the second was 107 patients with recurrent ischemic stroke. TTE was done for all patients. Both echocardiographic and clinical risk factors were compared between both groups. Logistic regression analysis identified predictors for recurrent strokes. Among clinical risk factors hypertension, hyperlipidemia, family history of atherosclerotic vascular disease, prior coronary artery disease, peripheral vascular disease, and chronic kidney disease were significantly higher in recurrent stroke group. Multivariate logistic analysis identified age (OR, 1.03; 95\% CI, 1.01 - 1.07), hypertension (OR, 2.25; 95\% CI, 1.03 - 4.92), and hyperlipidemia (OR, 2.73; 95\% CI, 1.40 - 5.35), as predictors for recurrent ischemic strokes. Left ventricular diastolic dysfunction, left ventricular hypertrophy (LVH) and aortic sclerosis were significantly more common in the recurrent stroke group compared to the first stroke group. However, in multivariate logistic analysis only LVH (OR, 3.50; 95\% CI, 1.69 - 7.23), was identified as a predictor for recur-

\footnotetext{
"Corresponding author.
}

rent strokes. Older age, hypertension, hyperlipidemia and left ventricular hypertrophy are significant predictors of recurrent ischemic strokes in patients with sinus rhythm. Those patients need more aggressive lipid lowering therapy and optimal blood pressure control.

\section{KEYWORDS}

Echocardiography; Risk Factors; Predictors; Ischemic Stroke

\section{INTRODUCTION}

Recurrent strokes are more likely to be disabling or fatal than first ever strokes [1]. Identifying characteristics of people at high risk of recurrence has important implications for planning secondary prevention strategies to reduce the disease burden. However, despite all efforts to reduce the risk of recurrence, $25 \%$ of stroke patients are reported to suffer from recurrent stroke [2]. The most relevant predictors of stroke recurrence identified in epidemiological trials include advancing age, arterial hypertension, atrial fibrillation, diabetes mellitus, hyperlipidemia $[3,4]$. However trials focusing on echocardiographic predictors for stroke recurrence are limited.

The goal of this study was to identify clinical and transthoracic echocardiographic predictors by comparing patients with first ischemic strokes and those with recurrent ischemic strokes. We also aimed to examine if echocardiographic risk stratification would be of additional value in predicting the recurrence of strokes compared with clinical predictors alone. 


\section{METHODS}

We conducted a prospective observational comparative study of 217 patients admitted to Mubarak Al-Kabeer hospital in Kuwait from January 2011, to January 2013, with the diagnosis of acute ischemic stroke and who were in sinus rhythm. Two groups of patients were simultaneously enrolled. The first group included $110 \mathrm{pa}-$ tients with first ischemic stroke and the second group included 107 patients with recurrent documented ischemic stroke. Informed consent was obtained from all patients.

All patients admitted with stroke were screened for inclusion in the study. Patients were not enrolled in the study if they had atrial fibrillation (AF) or poor TTE window. We tried to eliminate AF as known predictors for recurrent strokes [3,4], and because in patients with AF there is difficulty in accurately assessing important TTE parameters like diastolic dysfunction, also we have to take the average measurements for more than one cardiac cycle for each TTE parameter. Patients were also excluded if they developed an acute cardiac event after admission with stroke and before TTE was done as this would affect TTE findings. Other exclusion criteria included intracererbral bleeding of any type, hemorrhagic strokes, brain tumors, brain vascular lesions, or other brain masses, patients admitted with transient ischemic attack and those who refused to be enrolled in the study.

Ischemic stroke is defined by symptoms and rapidly developing signs of focal (or global) disturbance of cerebral function lasting more than 24 hours (unless interrupted by surgery or death), due to disturbance in the blood supply to the brain caused by blockage (thrombosis, arterial embolism) [5,6]. Recurrent stroke was defined as a new cerebrovascular event that met one of the following criteria: 1) the event resulted in a neurological deficit that was clearly different from that of the index stroke; 2) the event involved a anatomic site or vascular territory different from that of the index stroke; 3 ) the event was of a stroke subtype different from that of the index stroke $[7,8]$. The diagnosis of ischemic stroke was confirmed both by clinical presentation and neuroimaging. Patients were diagnosed clinically by their treating physician and the diagnosis was verified by a neurology specialist.

The basic demographic data and clinical cardiovascular risk factors were collected using a standardized case randomized form, including age, sex, smoking status (including shisha), hypertension, diabetes mellitus, hyperlipidemia, family history of atherosclerotic vascular diseases (coronary artery diseases, stroke, peripheral vascular disease) personal history of coronary artery disease, diagnosed peripheral vascular diseases and chronic kidney disease. All patients underwent brain computer tomography which confirmed the diagnosis of ischemic stroke. Patients with lacunar infarctions, large vessel infarctions or both were included in the study.

Complete TTE studies were done for all patients by two cardiology specialists not involved in patient treatment or care and all echocardiographic findings were collected using a standardized case randomized form. All normal and abnormal TTE findings were interpreted according to the recommendations of American Society of Echocardiography [9]. Both echocardiographic findings and clinical risk factors were compared between both patient groups. Other additional investigations were carried out at the discretion of the treating physician like carotid doppler imaging, transoesophageal echocardiography (TEE), magnetic resonance imaging, and magnetic resonance angiography.

\section{STATISTICAL ANALYSIS}

Data were collected and coded then entered into an IBM compatible computer, using the SPSS version 20 for Windows. Entered data were checked for accuracy then for normality, using Kolmogorov-Smirnov \& ShapiroWilk tests. Qualitative variables were expressed as numbers and percentages, while quantitative variables were expressed as means \pm standard deviation (SD). Independent samples t-test was used as a parametric test of significance for comparison between two sample means, after performing the Levene's test for equality of variances. The $\chi^{2}$-test (or likelihood ratio $=$ LLR) was used as a non-parametric test of significance for comparison between the distributions of two qualitative variables.

The Fisher's exact test was used as a non-parametric test of significance for comparison between the distributions of two qualitative variables whenever the $\chi^{2}$-test was not appropriate. Logistic regression model for factors significantly predicting the recurrence of stroke was also used. A 5\% level was chosen as a level of significance in all statistical significance tests used.

\section{RESULTS}

\subsection{Baseline Clinical Characteristics}

The study population included 217 patients (110 patients with first ischemic stroke and 107 patients with recurrent ischemic stroke) aged between 26 and 82 years. Mean age was $55 \pm 12$ years. The majority were men (155 patients, 71.4\%).

As regard the clinical risk factors in all the study population, 83 patients (38.2\%) were smokers, 120 patients (55.2\%) had diabetes mellitus, 163 patients $(75.1 \%)$ had hypertension, 140 patients (64.5\%) had hyperlipidemia, 50 patients (23\%) had family history of atherosclerotic vascular disease, 33 patients (15.2\%) had coronary artery disease, 5 patients (2.3\%) had diagnosed peripheral vas- 
cular disease and 15 patients (6.9\%) had chronic kidney disease.

Table 1 compares the baseline clinical characteristics between the two groups.

Hypertension was found in 70 patients (63.6\%) in first stroke group compared to 93 patients (86.9\%) in recurrent stroke group (OR, 3.79; 95\% CI $1.91-7.71 ; \mathrm{p}=$ 0.001 ), hyperlipidemia was found in 55 patients (50\%) in first stroke group compared to 85 patients $(79.4 \%)$ in recurrent stroke group (OR, 3.68; 95\% CI 2.12 - 7.03; p $=0.001$ ), family history of atherosclerotic vascular disease was found in 16 patients $(14.5 \%)$ in first stroke group compared to 34 patients $(31.8 \%)$ in recurrent stroke group (OR, 2.73; 95\% CI $1.40-5.33$; $\mathrm{p}=0.003$ ), coronary artery disease was found in 10 patients $(9.1 \%)$ in first stroke group compared to 23 patients (21.5\%) in recurrent stroke group (OR, 2.73; 95\% CI 1.23 - 6.07; p $=0.011$ ). Diagnosed peripheral vascular disease was absent in patients of first stroke group while present in five patients (4.7\%) in recurrent stroke group and chronic kidney disease was found in two patients (1.8\%) in first stroke group compared to 13 patients (21.1\%) in recurrent stroke group (OR, 7.46; 95\% CI 1.64 - 33.94; $\mathrm{p}=$ 0.003).

Despite the higher prevalence of diabetes mellitus in recurrent stroke group, in comparison with the first stroke group (61.7\% versus $49.1 \%$ respectively), no statistical significant difference was found between both groups ( $\mathrm{p}$ $=0.062$ ). Only history of smoking was higher in first stroke group, in 49 patients (44.5\%) compared to 34 patients (31.8\%) in recurrent stroke group but without statistical significant difference $(\mathrm{p}=0.053)$. Patients in first stroke group were significantly younger than in recurrent stroke group ( $52 \pm 12$ years versus $59 \pm 11$ years, $\mathrm{p}=$ $0.001)$.

\subsection{Echocardiographic Findings}

Abnormal TTE findings were found in 164 patients (75.6\%). TTE findings in both groups are summarized in (Table 2). Three abnormal TTE findings were signifi- cantly higher in the recurrent stroke group; namely Left ventricular diastolic dysfunction that was found in 42 patients (37.1\%) in first stroke group compared to 67 patients $(62.7 \%)$ in recurrent stroke group (OR, 2.71; 95\% CI 1.56 - 4.69; p = 0.001). LVH was found in 22 patients (20\%) in first stroke group compared to $53 \mathrm{pa}-$ tients (49.5\%) in recurrent stroke group (OR, 3.92; 95\% CI 2.15 - 7.16; $\mathrm{p}=0.001)$. Aortic sclerosis was found in 6 patients (5.5\%) in first stroke group compared to 15 patients (14\%) in recurrent stroke group (OR, 2.82; 95\% CI 1.05 - 7.58; $\mathrm{p}=0.003$ ).

The mean of ejection fraction in first group was $61 \% \pm$ $9 \%$ and was $59 \% \pm 10 \%$ for recurrent stroke group, however without statistical significant difference $(p=0.188)$. Despite the presence of other TTE abnormal findings in both groups such as LV systolic dysfunction in 16 patients (14.5\%) in first stroke group compared to 24 patients $(22.4 \%)$ in recurrent stroke group $(p=0.13)$, left atrial enlargement in 21 patients $(19 \%)$ in first stroke group compared to 28 patients $(26.1 \%)$ in recurrent stroke group $(\mathrm{p}=0.21)$, left ventricular dilatation in 8 patients (7.3\%) in first stroke group compared to 12 patients $(11.2 \%)$ in recurrent stroke group $(\mathrm{p}=0.31)$, regional wall motion abnormalities in 11 patients $(10 \%)$ in first stroke group compared to 15 patients (14\%) in prior stroke group ( $p=0.36)$, mitral regurgitation in 13 patients $(11.8 \%)$ in first stroke group compared to 18 patients $(16.8 \%)$ in recurrent stroke group $(p=0.29)$, tricuspid regurgitation in 9 patients $(8.2 \%)$ in first stroke group compared to 13 patients (12.2\%) in recurrent stroke group ( $\mathrm{p}=0.33$ ), LV Thrombus was found in one patient only in each group, none of these findings had significant statistical difference between both groups nor predict recurrent strokes.

In multivariate analysis, older age (OR $=1.03 ; 95 \%$ CI,1.01 - 1.07; $\mathrm{p}=0.002)$, hypertension (OR $=2.25 ; 95 \%$ $\mathrm{CI}, 1.03$ - 4.92; $\mathrm{p}=0.002)$ and hyperlipidemia $(\mathrm{OR}=$ 2.73; 95\% CI,1.07 - 5.35; $\mathrm{p}=0.002$ ), were statistically significant independent clinical predictors of recurrence of stroke (Table 3). Despite that the three abnormal TTE

Table 1. Demographic and clinical characteristics of study population.

\begin{tabular}{|c|c|c|c|}
\hline Characteristic & First ischemic stroke $(n=110)$ & Recurrent ischemic stroke $(n=107)$ & $\mathbf{P}$ \\
\hline Age (mean $\pm \mathrm{SD}$ (years) & $52 \pm 12$ & $59 \pm 11$ & 0.001 \\
\hline Male sex & $82(74.5 \%)$ & $73(68.2 \%)$ & 0.303 \\
\hline Smoking (including shisha) & $49(44.5 \%)$ & $34(31.8 \%)$ & 0.053 \\
\hline Diabetes mellitus & $54(49.1 \%)$ & $66(61.7 \%)$ & 0.062 \\
\hline Hypertension & $70(63.6 \%)$ & $93(86.9 \%)$ & 0.001 \\
\hline Dyslipidemia & $55(50 \%)$ & $85(79.4 \%)$ & 0.001 \\
\hline CAD & $10(9.1 \%)$ & $23(21.5 \%)$ & 0.011 \\
\hline PVD & $0(0 \%)$ & 5 (4.7\%) & 0.028 \\
\hline CKD & $2(1.8 \%)$ & $13(21.1 \%)$ & 0.003 \\
\hline
\end{tabular}

SD = Standard deviation, CAD = Coronary artery disease PVD = Peripheral vascular Disease, CKD = chronic kidney disease . 
Table 2. Echocardiographic findings of the study population.

\begin{tabular}{|c|c|c|c|}
\hline Echocardiographic Findings & First ischemic stroke $(n=110)$ & Recurrent ischemic stroke $(n=107)$ & $\mathbf{P}$ \\
\hline LVEF mean \pm SD \% & $61 \% \pm 9 \%$ & $59 \% \pm 10 \%$ & 0.188 \\
\hline left ventricular systolic dysfunction & $16(14.5 \%)$ & $24(22.4 \%)$ & 0.134 \\
\hline left ventricular diastolic dysfunction & $42(37.1 \%)$ & $67(62.7 \%)$ & 0.001 \\
\hline left ventricular hypertrophy & $22(20 \%)$ & $53(49.5 \%)$ & 0.001 \\
\hline Left ventricular dilatation & $8(7.3 \%)$ & $12(11.2 \%)$ & 0.316 \\
\hline Left atrial enlargement & $21(19 \%)$ & $28(26.1 \%)$ & 0.213 \\
\hline Right atrial enlargement & $0(0 \%)$ & $1(0.9 \%)$ & 0.493 \\
\hline Right ventricular enlargement & $0(0 \%)$ & $1(0.9 \%)$ & 0.493 \\
\hline Aortic sclerosis & $6(5.5 \%)$ & $15(14 \%)$ & 0.033 \\
\hline Aortic stenosis & $1(0.9 \%)$ & $6(5.6 \%)$ & 0.063 \\
\hline Aortic regurgitation & $8(7.3 \%)$ & $8(7.5 \%)$ & 0.954 \\
\hline Mitral stenosis & $1(0.9 \%)$ & $3(2.8 \%)$ & 0.365 \\
\hline Mitral valve prolapse & $0(0 \%)$ & $1(0.9 \%)$ & 0.493 \\
\hline Mitral regurgitation & $13(11.8 \%)$ & $18(16.8 \%)$ & 0.292 \\
\hline Tricuspid regurgitation & $9(8.2 \%)$ & $13(12.2 \%)$ & 0.333 \\
\hline Pulmonary hypertension & $6(5.5 \%)$ & $6(5.6 \%)$ & 0.961 \\
\hline Aortic dilatation & $1(0.9 \%)$ & $0(0 \%)$ & 1.000 \\
\hline Pericardial effusion & $1(0.9 \%)$ & $3(2.8 \%)$ & 0.365 \\
\hline Regional wall motion Abnormalities & $11(10 \%)$ & $15(14 \%)$ & 0.362 \\
\hline Atrial septal defect & $0(0 \%)$ & $0(0 \%)$ & - \\
\hline Patent foramen ovale & $3(2.7 \%)$ & $0(0 \%)$ & 0.247 \\
\hline Interatrial septal aneurysm & $2(1.8 \%)$ & $1(0.9 \%)$ & 1.000 \\
\hline Other cardiac defects & $1(0.9 \%)$ & $0(0 \%)$ & 1.000 \\
\hline Left ventricular thrombus & $1(0.9 \%)$ & $1(0.1 \%)$ & 1.000 \\
\hline Intracardiac masses & $0(0 \%)$ & $1(0.9 \%)$ & 0.129 \\
\hline
\end{tabular}

$\mathrm{LVEF}=$ left ventricular ejection fraction, $\mathrm{SD}=$ Standard deviation.

Table 3. Logistic regression model for factors predicting the recurrence of stroke.

\begin{tabular}{ccc}
\hline Predictors & Odds ratio & $\mathbf{9 5 \% ~ C I ~}$ \\
\hline Age & $1.03^{*}$ & $(1.01-1.07)$ \\
Hypertension & $2.25^{*}$ & $(1.03-4.92)$ \\
Hyperlipidemia & $2.73^{*}$ & $(1.40-5.35)$ \\
left ventricular hypertrophy & $3.50^{*}$ & $(1.69-7.23)$ \\
left ventricular diastolic dysfunction & 1.42 & $(0.74-2.76)$ \\
Aortic sclerosis & 3.30 & $(0.37-29.61)$ \\
Left ventricular dilatation & 1.88 & $(0.53-6.63)$ \\
left ventricular systolic dysfunction & 0.99 & $(0.95-1.02)$ \\
Left atrial enlargement & 0.62 & $(0.27-1.39)$ \\
\hline
\end{tabular}

* $=$ significant at $5 \%$ level.

findings; namely LV diastolic dysfunction, LVH and Aortic sclerosis, using bivariate analysis, were significantly higher in prior stroke group, only LVH in multivariate analysis $(\mathrm{OR}=3.5$; 95\% CI, $1.69-7.23$; $\mathrm{p}=$ 0.002 ), was the significant echocardiographic predictor for recurrence of strokes (Table 3). It should be noted that all other variables entered in this model were not significant predictors for recurrence of strokes. These are left ventricular diastolic dysfunction, Aortic sclerosis, Left ventricular dilatation, left ventricular systolic dysfunction, and Left atrial enlargement ( $\mathrm{p}$-values were > $0.05)$.

\section{DISCUSSION}

In this study three TTE abnormalities (LV diastolic dysfunction, LVH and aortic sclerosis) were significantly higher in recurrent stroke group than in those with first stroke group. Adjusted analysis showed only LVH was a predictor for recurrence of ischemic strokes.

Transthoracic echocardiography is part of the routine workup of stroke patients in many centers across the world, to look for a cardiac source of embolus [10,11]. However, there is an ongoing debate regarding the cost effectiveness of this diagnostic tool. Most studies on the role of echocardiography on the management of acute stroke patients had focused on the capacity of TTE or transesophageal echocardiography to find potential sources of embolism [12-14]. Although cardioembolism might be responsible for $15 \%$ to $20 \%$ of ischemic strokes, the majority of patients undergoing TTE had no findings sug- 
gestive of cardioembolism [15]. A recent hospital-based study from Canada reported that TTE has a low yield, diagnosing only $4 \%$ of ischemic stroke patients with abnormalities suggesting cardioembolic etiology [16]. Other cardiac structural and functional abnormalities that had been associated with cardioembolic stroke include LA dilatation, poor LV systolic function, valvular heart diseases, cardiac tumors, patent foramen ovale and atrial septal defect [17-19]. Instead of just focusing on finding potential sources of embolism, our study focused on finding other additional abnormalities that may help predict recurrence of ischemic strokes with difference between the two focuses.

In our study three in four patients had abnormal TTE findings. From these findings LV systolic dysfunction, LA enlargement, LV dilatation, regional wall motion abnormalities and mitral regurgitation. LV systolic dysfunction was found in 16 patients (14.5\%) in first stroke group compared to 24 patients (22.4\%) in recurrent stroke group ( $\mathrm{P}=0.13)$, left atrial (LA) enlargement was found in 21 patients (19\%) in first stroke group compared to 28 patients (26.1\%) in recurrent stroke group ( $\mathrm{P}=0.21)$, left ventricular dilatation was found in 8 patients $(7.3 \%)$ in first stroke group compared to 12 patients (11.2\%) in recurrent stroke group $(\mathrm{P}=0.31)$, regional wall motion abnormalities was found in 11 patients $(10 \%)$ in first stroke group compared to 15 patients (14\%) in recurrent stroke group $(\mathrm{P}=0.36)$, mitral regurgitation was found in 13 patients (11.8\%) in first stroke group compared to 18 patients $(16.8 \%)$ in recurrent stroke group $(\mathrm{P}=0.29)$, none of these findings had significant statistical difference between both groups nor predict recurrent strokes.

In another study LV systolic dysfunction was a powerful, consistent, and independent predictor of stroke in patients with AF despite neither LA diameter nor mitral regurgitation was significantly associated with risk of stroke in this study [20]. In the study of Gambini and Paciaroni, LA diameter was significantly associated with the combined end point of ischemic stroke and systemic emboli [21]. However, it was not predictive of stroke in another larger cohort of patients with AF given aspirin in the SPAF study [22]. In both these studies, LV dysfunction emerged as a powerful independent risk factor for stroke[21,22].

In our study population group $38.2 \%$ were smokers, 55.29\% had diabetes mellitus, 75.1\% had hypertension, and $64.5 \%$ had hyperlipidemia. Among these risk factors only older age, hypertension and hyperlipidemia were significant clinical predictors for recurrence of strokes. The most relevant predictors of stroke recurrence identified in epidemiological trials include advancing age, arterial hypertension, atrial fibrillation, diabetes mellitus, hyperlipidemia [3,4]. A previous study suggested that clinical risk factors as hypertension, diabetes mellitus, hyperlipidemia, ischemic heart disease, atrial fibrillation, smoking and carotid stenosis were contributing factors for stroke disease [23]. However, the minor differences in the prevalence of stroke risk factors in different communities are probably due to differences in culture, disease patterns, living habits and distribution of various ethnic groups. The clinical factors that were independently associated with stroke in the analysis of patients in a prospective study of patients from three clinical trials with full echocardiographic evaluations were increasing age, previous TIA or stroke, history of diabetes, or history of heart failure [20]. In agreement with our study, other studies have shown that all the above risk factors are more prevalent among male subjects [24-26]. Similar to our study, older studies showed that stroke prevalence rises with older age group [27]. With advancing age, the effect of increasingly high frequency of contributing factors such as hypertension, diabetes and hyperlipidemia might be a reason for the increased prevalence of stroke $[24,28]$.

In our study hypertension was the first common risk factor as was present in $75.1 \%$ of our patients. Valery et al from Russia found $84.8 \%$ of their patients had hypertension [29]. Ong and Raymond from Singapore found $71.5 \%$ of their patients had hypertension [30]. In our study hyperlipidemia was the second most common risk factor, as was present in $64.5 \%$ of our patients with significant higher prevalence in recurrent stroke group, and this was higher than that reported in other studies [23]. In our study diabetes was the third most common risk factor as was present in $55.2 \%$ of all patient with significant higher prevalence in recurrent stroke group, this was also higher than reported in other industrialized countries where varied from $7 \%$ to $41 \%$, in addition, researchers believed that diabetic patients are at an increased risk for hypertension and stroke [30,31].

In our study $38.2 \%$ were smoker and was higher in first stroke group who are younger than in recurrent stroke group which may furthermore contribute to uncommon etiologies in younger patients. The mechanisms by which cigarette smoking is thought to increase the likelihood of ischemic stroke include increased fibrinogen levels, platelet adhesiveness and reduced cerebral blood flow due mainly to atheroma formation associated with smoking and higher blood viscosity in chronic smokers [32,33]. The main limitation of this study was the relatively small number of patients examined and TTE findings were not corrected for body surface area which was difficult to assess due to difficult mobility in stroke patients with residual neurological deficits and disabilities.

\section{CONCLUSION}

Transthoracic echocardiography is a valid diagnostic modality for stroke workup and should be considered as an 
essential test in all ischemic stroke patients. In patients with recurrent ischemic strokes who have sinus rhythm, among TTE abnormalities only LVH was a significant predictor for recurrence of ischemic strokes. Patients with recurrent ischemic strokes tended to be older with more clinical risk factors, among which aging, hypertension and hyperlipidemia were predictors for recurrent ischemic strokes. Those patients need more aggressive lipid lowering therapy and optimal control of blood pressure. Further studies with a larger sample size are needed to provide more supporting results to our study.

\section{REFERENCES}

[1] Bonita, R. (1992) Epidemiology of stroke. Lancet, 339, 342-344. http://dx.doi.org/10.1016/0140-6736(92)91658-U

[2] Hardie, K., Jamrozik, K., Hankey, G.J., Broadhurst, R.J. and Anderson, C. (2005) Trends in five-year survival and risk of recurrent stroke after first-ever stroke in the Perth Community Stroke Study. Cerebrovascular Diseases, 19, 179-185. http://dx.doi.org/10.1159/000083253

[3] Sacco, R.L., Shi, T., Zamanillo, M.C. and Kargman, D.E. (1994) Predictors of mortality and recurrence after hospitalized cerebral infarction in an urban community: The northern manhattan stroke study. Neurology, 44, 626-634. http://dx.doi.org/10.1212/WNL.44.4.626

[4] Leoo, T., Lindgren, A., Petersson, J. and von Arbin, M. (2008) Risk Factors and Treatment at Recurrent Stroke Onset: Results from the Recurrent Stroke Quality and Epidemiology (RESQUE) Study. Cerebrovascular Diseases, 25, 254-260. http://dx.doi.org/10.1159/000113864

[5] Feinberg, W.M., Blackshear, J.L., Laupacis, A., Kronmal, R. and Hart, R.D. (1995) Prevalence, age distribution, and gender of patients with atrial fibrillation. Analysis and implications. JAMA Internal Medicine, 155, 469-473. http://dx.doi.org/10.1001/archinte.1995.00430050045005

[6] Donnan, G.A., Fisher, M., Macleod, M. and Davis, S.M. (2008) Stroke. Lancet, 371, 1612-1623. http://dx.doi.org/10.1016/S0140-6736(08)60694-7

[7] Sacco, R.L., Foulkes, M.A., Mohr, J.P., Wolf, P.A., Hier, D.B. and Price, T.R. (1989) Determinants of early recurrence of cerebral infarction: The Stroke Data Bank. Stroke, 20, 983-989. http://dx.doi.org/10.1161/01.STR.20.8.983

[8] Hier, D.B., Foulkes, M.A., Swiontoniowski, M., Sacco, R.L., Gorelick, P.B., Mohr, J.P., et al. (1991) Stroke recurrence within 2 years after ischemic infarction. Stroke, 22, 155-161. http://dx.doi.org/10.1161/01.STR.22.2.155

[9] Lang, R.M., Bierig, F.M., Devereux, R.B., Flachskampf, F.A., Foster, E., Pellikka, P.A., et al. (2005) Recommendations for chamber quantification: A report from the American Society of Echocardiography's Guidelines and Standards Committee and the Chamber Quantification Writing Group. Journal of the American Society of Echocardiography, 18, 1440-1463.

http://dx.doi.org/10.1016/j.echo.2005.10.005
[10] Goldstein, L.B., Matchar, D.B., Hoff-Lindquist, J., Samsa, G.P. and Homer, R.D. (2003) Veterans Administration Acute Stroke (VASt) Study: Lack of race/ethnic-based differences in utilization of stroke-related procedures or services. Stroke, 34, 999-1004. http://dx.doi.org/10.1161/01.STR.0000063364.88309.27

[11] Cereda, M. and Trocino, G. (2002) Echocardiography in patients with acute cerebrovascular syndromes. Rational use and clinical impact. Italian heart journal. Supplement, 3, 26-35.

[12] Blum, A., Reisner, S. and Farbstein, Y. (2004) Transesophageal echocardiography (TEE) vs. transthoracic echocardiography (TTE) in assessing cardiovascular sources of emboli in patients with acute ischemic stroke. Medical Science Monitor, 10, CR521-CR523.

[13] Leung, D.Y., Black, I.W., Cranney, G.B., Walsh, W.F., Grimm RA, Stewart WJ, et al. (1995) Selection of patients for transesophageal echocardiography after stroke and systemic embolic events: role of transthoracic echocardiography. Stroke, 26, 1820-1824. http://dx.doi.org/10.1161/01.STR.26.10.1820

[14] Gambini, C. and Paciaroni, E. (1995) The role of transesophageal echocardiography in the diagnosis of ischemic stroke in the elderly. Archives of Gerontology and Geriatrics, 20, 37-42. http://dx.doi.org/10.1016/0167-4943(94)00603-5

[15] Semple, P.F. and Sacco, R.L. (1999) An Atlas of Stroke. 2nd Edition, the Parthenon Publishing Group, London, 24.

[16] Douen, A., Pageau, N. and Medic, S. (2007) Usefulness of cardiovascular investigations in stroke management. Clinical relevance and economic implications. Stroke, $\mathbf{3 8}$, 1956-58. http://dx.doi.org/10.1161/STROKEAHA.106.477760

[17] Albers, G.W., Amarenco, P., Easton, J.D., Sacco, R.L. and Teal, P. (2004) Antithrombotic and thrombolytic therapy for ischemic stroke: The seventh ACCP conference on antithrombotic and thrombolytic therapy. Chest, 126, S483-S512.

[18] Nalluri, C., Jain, N., Dalton, N.D., Fox, K. and Hoit, B.D. (2002) The role of transesophageal echocardiography in the evaluation of embolic stroke: Is management altered. Cardiac Ultrasound Today, 8, 97-117.

[19] Rem, J.A., Hachinski, V.C., Boughner, D.R. and Bemett, H.J. (1985) Value of cardiac monitoring and echocardiography in TIA and stroke patients. Stroke, 16, 950-956. http://dx.doi.org/10.1161/01.STR.16.6.950

[20] Ezekowitz, M., Laupacis, A., Boysen, G., Connolly, S., Hart, R., James, K., et al. (1998) Echocardiographic predictors of stroke in patients with atrial fibrillation. A prospective study of 1066 patients from 3 clinical trials. JAMA Internal Medicine, 158, 1316-1320. http://dx.doi.org/10.1001/archinte.158.12.1316

[21] SPAF Investigators. (1992) Predictors of thromboembolism in atrial fibrillation, II: Echocardiographic features of patients at risk-the stroke prevention in atrial fibrillation investigators. Annals of Internal Medicine, 116, 6-12. http://dx.doi.org/10.7326/0003-4819-116-1-6

[22] SPAF Investigators. (1995) Risk factors for thromboem- 
bolism during aspirin therapy in patients with atrial fibrillation. Journal of Stroke and Cerebrovascular Diseases, 5, 147-157. http://dx.doi.org/10.1016/S1052-3057(10)80166-1

[23] Barech, M.S., Sadiq, S.M., Zarkoon, A.K. and Gulandam, K. (2010) Risk factors for ischemic stroke in patients attending a tertiary hospital in Quetta. Pakistan Journal of Neurological Sciences, 5, 1-5.

[24] Ayala, C., Croft, J.B., Greenlund, K.J., Keenan, N.L., Donehoo, R.S., Malarcher, A.M., et al. (2002) Sex differences in US mortality rates for stroke and stroke subtypes by race/ethnicity and age, 1995-1998. Stroke, 33, $1197-1201$.

http://dx.doi.org/10.1161/01.STR.0000015028.52771.D1

[25] Peter, A., Birgitta, S. and Andreas, T. (2009) Sex differences in stroke epidemiology: A systematic review. Stroke, 40, 1082-1090. http://dx.doi.org/10.1161/STROKEAHA.108.540781

[26] Eyal, S., Lloyd, E.C., Wayne, D.R., Lori, L.B., Christie, M.B., Paul, G.M., et al. (2003) Plasma lipid profile and incident ischemic stroke: The atherosclerosis risk in communities (ARIC) study. Stroke, 34, 623-631. http://dx.doi.org/10.1161/01.STR.0000057812.51734.FF

[27] David, J.C., Robert, D.A., Charles, J.M., Beatriz, L.R., Cecil, M.B., Dan, S.S., et al. (1996) Age-related changes in stroke risk in men with hypertension and normal blood pressure. Stroke, 27, 819-824. http://dx.doi.org/10.1161/01.STR.27.5.819
[28] Toshio, H., Seinosuke, K., Hideki, I., Nobuhiro, Y., Hirohito, S., Hircshi, W., et al. (2009) Low HDL cholesterol is associated with the risk of stroke in elderly diabetic individuals. Diabetes Care, 32, 1221-23. http://dx.doi.org/10.2337/dc08-1677

[29] Valery, L.F., David, O.W., Yury, P.N., Michael, W.F. and Jack, P.W. (1998) Risk factors for ischemic stroke in a Russian community a population-based case-control study. Stroke, 29, 34-39. http://dx.doi.org/10.1161/01.STR.29.1.34

[30] Ong, T.Z. and Raymond, A.A. (2002) Risk factors for stroke and predictors of one-month mortality. Singapore Medical Journal, 43, 517-521.

[31] Brett, M.K., Jane, K., Dawn, K., Daniel, A., Alexander, S., Kathleen A, et al. (2005) Epidemiology of ischemic stroke in patients with diabetes. Diabetes Care, 28, 355359. http://dx.doi.org/10.2337/diacare.28.2.355

[32] Tanika, N.K., Gu, D.F., Jing, C., Huang, J.-F., Chen, J.-C., Duan, X.F., et al. (2008) Cigarette smoking and risk of stroke in the Chinese adult population. Stroke, 39, 16881693. http://dx.doi.org/10.1161/STROKEAHA.107.505305

[33] Jeroen, N.S., van Genugten, M.L.L., Silivia, M.A., Andre, J.A., Caroline, A.B., et al. (2005) Modeling the future burden of stroke in the Netherlands impact of aging, smoking, and hypertension. Stroke, 36, 1648-1655. http://dx.doi.org/10.1161/01.STR.0000173221.37568.d2 